\title{
Statistical Analyses of Random Telegraph Noise in Pixel Source Follower with Various Gate Shapes in CMOS Image Sensor
}

\author{
Shinya Ichino ${ }^{\dagger}$, Takezo Mawaki ${ }^{\dagger}$, Akinobu Teramoto ${ }^{\dagger \dagger}$, Rihito Kuroda ${ }^{\dagger}$ (member), \\ Shunichi Wakashima ${ }^{\dagger}$, Tomoyuki Suwa ${ }^{\dagger \dagger}$ and Shigetoshi Sugawa ${ }^{\dagger, \dagger}$ (member)
}

\begin{abstract}
Random telegraph noise (RTN) that occurs at in-pixel source follower (SF) transistors and column amplifier is one of the most important issues in CMOS image sensors (CIS) and reducing RTN is a key to the further development of CIS. In this paper, we clarified the influence of transistor shapes on RTN from statistical analysis of SF transistors with various gate shapes including rectangular, trapezoidal and octagonal structures by using an array test circuit. From the analysis of RTN parameter such as amplitude and the current-voltage characteristics by the measurement of a large number of transistors, the influence of shallow trench isolation (STI) edge on channel carriers and the influence of the trap location along source-drain direction are discussed by using the octagonal SF transistors which have no STI edge and the trapezoidal SF transistors which have an asymmetry gate width at source and drain side.
\end{abstract}

Keywords: CMOS image sensor, in-pixel source follower, random telegraph noise, shallow trench isolation, transistor shape.

\section{Introduction}

Random telegraph noise (RTN) is one of the low frequency noise which occurs in MOSFETs, and the magnitude of RTN cannot be negligible as the downscaling of CMOS devices continues ${ }^{1}$. Recently, it is considered that RTN has been one of the most important issues in CMOS image sensors (CIS) to achieve high resolution and high sensitivity in CIS. In high sensitivity CIS, RTN that occurs at in-pixel source follower (SF) transistors and column amplifier causes the deterioration of image quality especially in low illuminance, and RTN is one of the major limiting factors of the dark random noise ${ }^{2-4)}$. Therefore, reducing RTN is a key to the further development of CIS.

In order to find out the method of reducing $\mathrm{RTN}$, the RTN of SF transistors with new transistor structure and process technology has been investigated by using an array test circuit. For instance, it has been reported that buried channel transistor ${ }^{5)-7)}$, atomically flat gate insulator film ${ }^{8)}$ and ring shaped transistor ${ }^{9)-11)}$ without

Received December 20, 2017; Revised March 14, 2018; Accepted April 24, 2018

$\dagger$ Graduate School of Engineering, Tohoku University (Sendai, Japan)

$\dagger+$ New Industry Creation Hatchery Center, Tohoku University (Sendai, Japan) shallow trench isolation (STI) are effective. Furthermore, it has been also reported that behaviors of RTN depend on the SF operating conditions such as drain current $\left(I_{\mathrm{DS}}\right)$, back bias $\left(V_{\mathrm{BS}}\right)$ and measured temperature ${ }^{12)-15)}$.

It is believed that the origin of RTN is random capture or emission of carriers by or from traps near the gate insulator film and Si interface ${ }^{16)}$. These phenomena cause a discrete and stochastic fluctuation in drain current or threshold voltage $\left(V_{\text {th }}\right)$ in two or more states $^{17)}$. In order to evaluate the influence of RTN in CIS, the statistical analysis of the appearance probability and the magnitude of RTN are important. Although the magnitude of RTN is generally characterized by the root mean square (RMS) of the output voltage, it has been reported that the amplitude of RTN which is the difference of voltage between high state and low state is an important parameter characterizing the statistical noise distribution ${ }^{15}$.

This paper is the extended version of the conference presentation (2017 IISW), which reported the reduction effect of RTN from the consideration of the multiple aspects such as statistical analysis of RTN parameters and currentvoltage characteristics by the measurement of a large number of the transistors with various gate shapes by means of a low noise array test circuit measurement system with the floor noise of $35 \mu V_{\mathrm{RMS}^{11)}}{ }^{15)}$. In addition to 
conventional rectangular SF transistors, the trapezoidal SF transistors which have an asymmetric gate width at source and drain side, and octagonal SF transistors which has no STI edge were also measured and analyzed using the same evaluation system. In this paper, we additionally analyzed current-voltage characteristics and RTN parameters in detail with wider range of drain current. From the obtained results, we discuss the gate shape of pixel source follower effective for reducing RTN in CIS.

\section{Experimental method}

Figure 1 shows the circuit schematic diagram and the chip micrograph of the array test circuit used for the evaluation in this paper. Table I shows its specifications. The array test circuit was fabricated by a 1 poly-Si 5 metal $0.18 \mu \mathrm{m}$ CMOS technology with pinned photodiode (PD) and STI isolation. It consists of pixel arrays including measured SF transistors, column current sources, analog memories, output buffers and two shift registers (vertical and horizontal). The unit pixel was fabricated with a structure in which second reset gate $(R)$ was added to a conventional four transistor pixel of CIS. In this work, SF output signal was obtained by applying a constant voltage directly from the reset voltage node (VR) to floating diffusion (FD) node. The drain current of measured SF transistor was set by the column current sources connected to SF output lines. There are two readout paths used for different measurement modes in the column readout circuits. One path is connected to analog memories similar to conventional CIS with analog signal readout. The data of output signal of a large number of transistors can be obtained in short time by using this path. The other path is directly connected to the chip output buffer for high speed continuous signal sampling for selected pixels by two shift registers. In this work, the latter path was used for the detailed RTN parameter analysis from output signal waveform of time domain.

Table II shows the specifications of the measured SF transistor in pixel arrays. In this work, trapezoidal and octagonal transistors were also measured in addition to conventional rectangular transistors as shown in Fig. 2. Trapezoidal transistors (Pix. 5 and 6) have an asymmetric gate width at source and drain side, and the gate width of the wider side is about three times wider than that of the narrower side. They are to be effective for the evaluation of the influence of the trap location along source-drain direction ${ }^{18)}$. Octagonal transistors

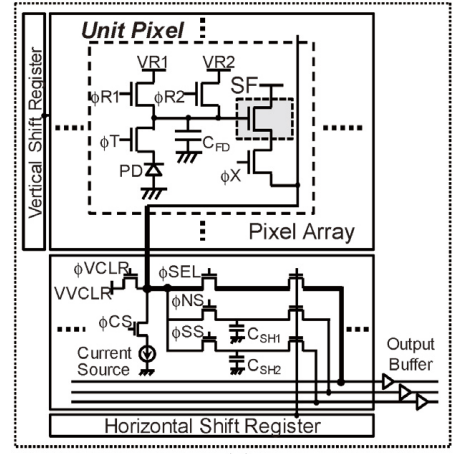

(a)

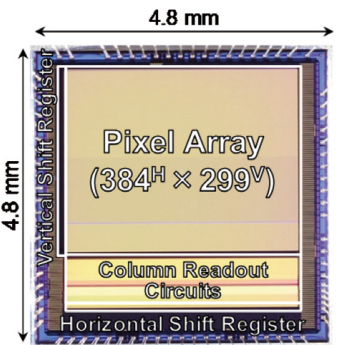

(b)
Fig. 1 (a) Circuit schematic diagram and (b) chip micrograph of the array test circuit used for the evaluation in this paper.

Table I Array test circuit specifications.

\begin{tabular}{cc}
\hline Process technology & 1 poly-Si5 metal 0.18 $\mu \mathrm{m}$ \\
CMOS with pinned-PD $(3.3 \mathrm{~V})$ \\
Die size & $4800 \mathrm{~m}^{\mathrm{H}} \times 4800 \mathrm{~m}^{\mathrm{V}}$ \\
Pixel size & $10 \mu \mathrm{m}^{\mathrm{H}} \times 10 \mu \mathrm{m}^{\mathrm{V}}$ \\
Tox & $7.8 \mathrm{~nm}$ \\
SF channel type & Buried channelnMOSFET \\
\hline
\end{tabular}

Table II Specifications of the measured SF transistors in pixel arrays.

\begin{tabular}{|c|c|c|c|c|c|c|}
\hline $\begin{array}{l}\text { Pixel } \\
\text { No. }\end{array}$ & SF shape & $\begin{array}{c}W \\
{[\mu \mathrm{m}]}\end{array}$ & $\begin{array}{c}\mathrm{L} \\
{[\mu \mathrm{m}]}\end{array}$ & $\mathrm{W} / \mathrm{L}$ & $\begin{array}{l}\text { Area } \\
{\left[\mathrm{\mu m}^{2}\right]}\end{array}$ & $\begin{array}{l}\text { Number of } \\
\text { transistors }\end{array}$ \\
\hline Pix. 1 & \multirow{4}{*}{ Rectangle } & 0.34 & 0.52 & 0.65 & 0.177 & 4608 \\
\hline Pix. 2 & & 0.66 & 0.52 & 1.27 & 0.343 & 4608 \\
\hline Pix. 3 & & 1.36 & 0.52 & 2.62 & 0.707 & 4608 \\
\hline Pix. 4 & & 3.27 & 0.52 & 6.29 & 1.700 & 4608 \\
\hline Pix. 5 & \multirow{2}{*}{ Trapezoid } & $\begin{array}{c}\mathrm{W}_{\text {Ave }}: 0.66 \\
\mathrm{~W}_{\mathrm{S}} / \mathrm{W}_{\mathrm{D}}: 0.98 / 0.34\end{array}$ & 0.52 & 1.27 & 0.343 & 9216 \\
\hline Pix. 6 & & $\begin{array}{c}\mathrm{W}_{\text {Ave }}: 0.66 \\
\mathrm{~W}_{\mathrm{S}} / \mathrm{W}_{\mathrm{D}}: 0.34 / 0.98 \\
\end{array}$ & 0.52 & 1.27 & 0.343 & 4608 \\
\hline Pix. 7 & Octagon & $\begin{array}{c}\mathrm{W}_{\text {Ave }}: 3.78 \\
\mathrm{~W}_{\mathrm{S}} / \mathrm{W}_{\mathrm{D}}: 5.51 / 2.06\end{array}$ & 0.52 & 7.27 & 1.966 & 4608 \\
\hline
\end{tabular}
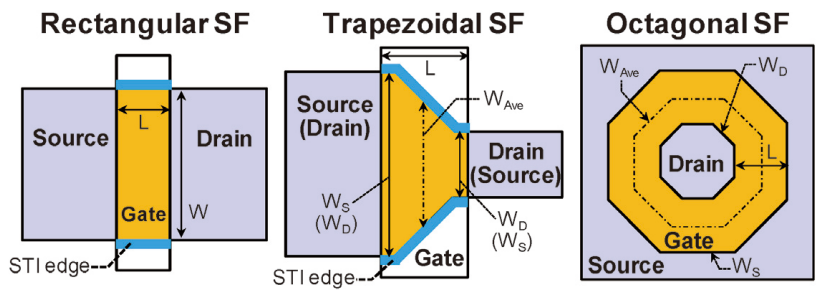

Fig. 2 Layout diagrams of the SF transistors with different types of shape, rectangular, trapezoidal and octagonal SF.

(Pix. 7) have no STI edge similar to the ring shaped transistor ${ }^{9)-11)}$. They are to be effective for the evaluation of the influence of the STI edge on channel carriers. Here, Pix. 4 is the rectangular transistor with nearly the same gate area as the octagonal transistor. All of the transistor types have a common gate length of $0.52 \mu \mathrm{m}$. The measured 4608 (9216 in Pix. 5) SF transistors are buried channel which is known to be effective for reducing $\mathrm{RTN}^{5)-7)}$. The measurement conditions were summarized in Table III. The SF drain current was 
Table III Measurement conditions of array test circuit.

\begin{tabular}{cc}
\hline SF drain current & $0.1 \mu \mathrm{A}-11 \mu \mathrm{A}$ \\
Sampling period & $1 \mu \mathrm{s}$ \\
Sampling time & $0.1 \mathrm{~s}$ \\
(Sampling point) & $(100000$ points) \\
Temperature & $25^{\circ} \mathrm{C}$ \\
\hline
\end{tabular}

applied from $0.1 \mu \mathrm{A}$ to $11 \mu \mathrm{A}$, where it is comparable value of drain current for typical pixel readout operation in CIS (several $\mu \mathrm{A}$ to about $10 \mu \mathrm{A}$ ).

\section{Results and discussion}

In this paper, the evaluation of the influence of STI edge on channel carriers using the octagonal SF transistors and the evaluation of the influence of trap location along source-drain direction using the trapezoidal SF transistors are discussed. At first, the comparison between the octagonal SF transistors and the rectangular SF transistors with nearly the same gate area is discussed. Figure 3 shows the cumulative probability of $V_{\text {RMS }}$ in the octagonal and the rectangular SF transistors in the Gumbel plot $\left(I_{\mathrm{DS}}=0.1 \mu \mathrm{A}\right.$ and $\left.1 \mu \mathrm{A}\right)$. $V_{\mathrm{RMS}}$ in this work indicates the input referred value at FD node. The $\mathrm{SF}$ output voltages were set at $1.9 \mathrm{~V}$ for each type of transistor in order to equalize the influence of back bias for all measured pixels. From the distribution in Fig. 3, the octagonal $\mathrm{SF}$ transistors have smaller $V_{\mathrm{RMS}}$ values than that of the rectangular SF transistors under the constant drain current condition. Figure 4 shows $V_{\text {RMS }}$ values of the octagonal and the rectangular SF transistors at the cumulative probability of $99.9 \%$ as a function of the drain current. $V_{\mathrm{RMS}}$ of both types are nearly equal when the drain current is large. However, as the drain current decreases, the $V_{\text {RMS }}$ becomes larger in the rectangular SF transistors and the difference of $V_{\text {RMS }}$ between the octagonal and the rectangular SF transistors appears obviously. Figure 5 shows the $I_{\mathrm{DS}^{-}}$

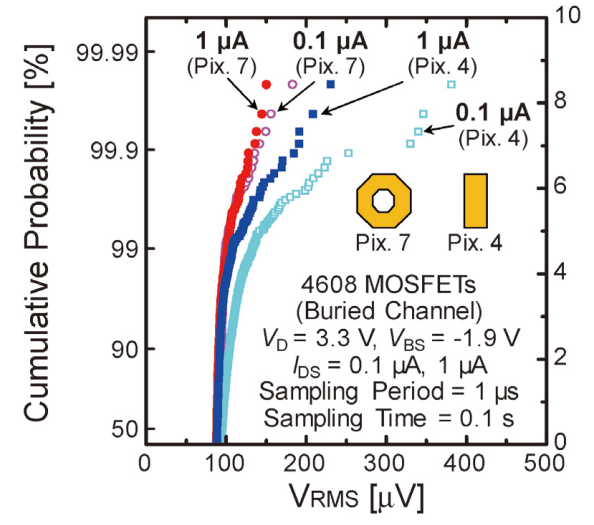

Fig. 3 Cumulative probability of $V_{\text {RMS }}$ in the Gumbel plot (Pix. 4 and 7). The comparison between rectangular and octagonal $\mathrm{SF}$ transistors with nearly equal gate area under the constant drain current condition $\left(I_{\mathrm{DS}}=0.1 \mu \mathrm{A}\right.$ and $\left.1 \mu \mathrm{A}\right)$ is shown.

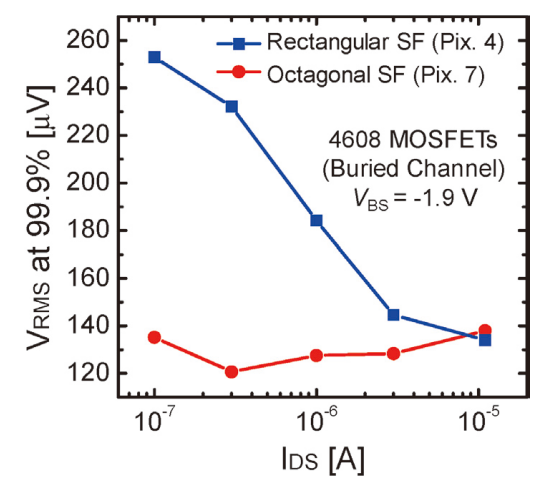

Fig. $4 V_{\text {RMS }}$ at the cumulative probability of $99.9 \%$ as a function of drain current (Pix. 4 and 7).

$V_{\mathrm{GS}}$ curves of 4608 rectangular and octagonal SF transistors, respectively. It is clear that in the octagonal SF transistors, the variation of $V_{\mathrm{GS}}$ at low drain current condition is smaller than that of the rectangular SF transistors and the slopes of $I_{\mathrm{DS}}-V_{\mathrm{GS}}$ curves in subthreshold region are steeper than those of the rectangular SF transistors. Figure 6 shows the appearance probability histogram of subthreshold swing (SS) of the octagonal and the rectangular SF transistors
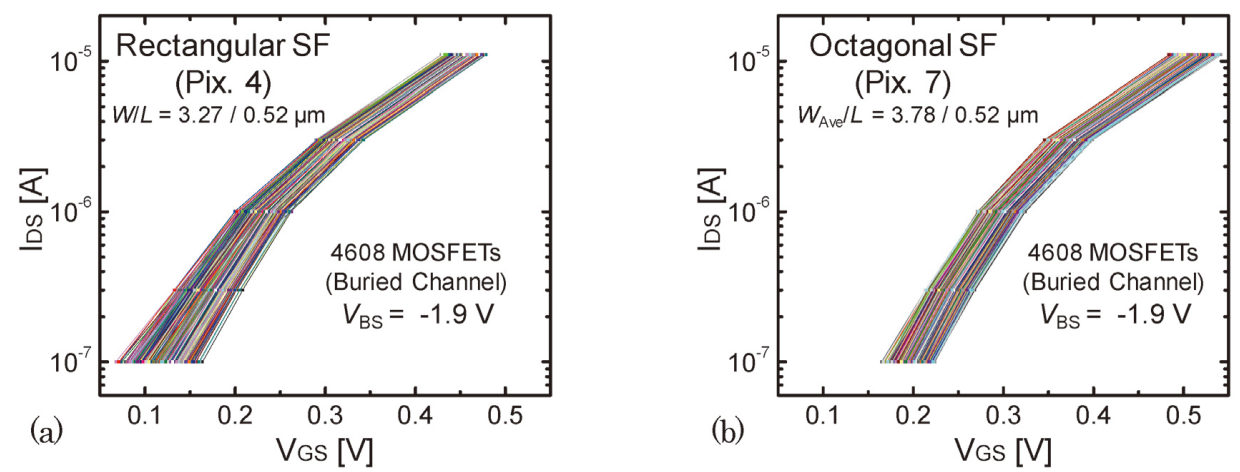

Fig. $5 I_{\mathrm{DS}}-V_{\mathrm{GS}}$ curves of all measured SF transistors (4608 pixels). (a) Rectangular (Pix. 4) and (b) octagonal SF transistors (Pix. 7). The output voltages were set at $1.9 \mathrm{~V}$ for each type of SF transistor. 


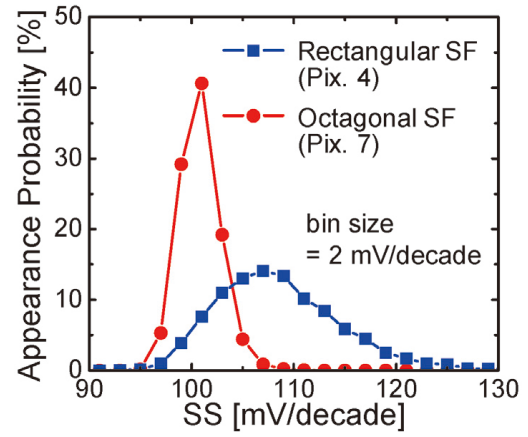

Fig. 6 Appearance probability histogram of SS (Pix. 4 and 7). SS was extracted from $I_{\mathrm{DS}}$ region of $10^{-7} \mathrm{~A}$ to $10^{-6} \mathrm{~A}$ for each type of SF transistor.

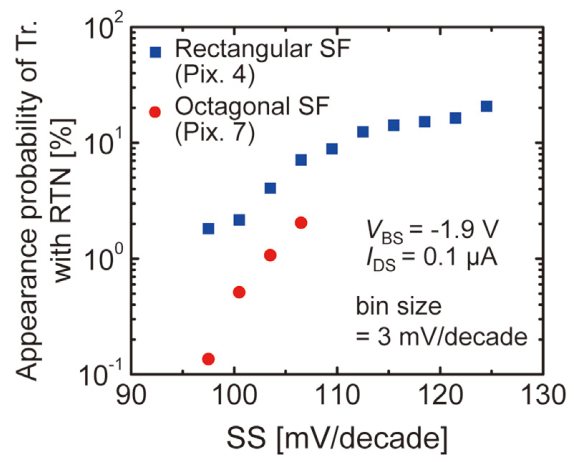

Fig. 7 Appearance probability of SF transistors with RTN as a function of SS (Pix. 4 and 7).

extracted from the $I_{\mathrm{DS}}-V_{\mathrm{GS}}$ curves in Fig. 5. In this work, SS was extracted from $I_{\mathrm{DS}}$ region of $10^{-7} \mathrm{~A}$ to $10^{-6} \mathrm{~A}$ for each type of the SF transistor. The SS and the variation of SS in the octagonal SF transistors were smaller than those of the rectangular SF transistors. Figure 7 shows the appearance probability of SF transistors with RTN calculated for each bin as a function of the segmented SS regions for the octagonal and the rectangular SF transistors. Here, the bin size of SS was taken at 3 mV/decade, and a pixel showing $V_{\text {RMS }}$ of more than 105 $\mu \mathrm{V}$ and amplitude of more than $130 \mu \mathrm{V}$ was defined as an RTN appearing pixel from the consideration of the $V_{\text {RMS }}$ distribution shown in Fig. 3. The appearance probability of SF transistors with RTN increases with SS increasing as previously reported ${ }^{19)}$. Furthermore, the appearance probability of RTN in the rectangular SF transistors is larger than that of the octagonal SF transistors at the same range of SS.

Figure 8 shows the standard deviation of $V_{\mathrm{GS}}$ in the octagonal and the rectangular SF transistors extracted from the $I_{\mathrm{DS}}-V_{\mathrm{GS}}$ curves in Fig. 5 as a function of the drain current. The $\sigma V_{\mathrm{GS}}$ became larger in the rectangular SF transistors when the drain current decreases, whereas it did not change significantly in the octagonal SF transistors. Figure 9 shows the standard deviation of $V_{\mathrm{GS}}$ in the octagonal and various

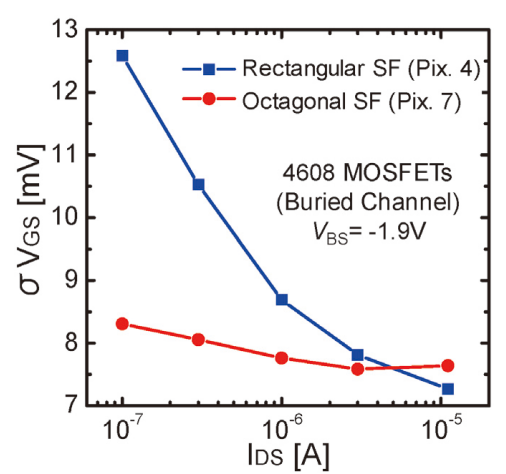

Fig. 8 Standard deviation of $V_{\mathrm{GS}}$ extracted from $I_{\mathrm{DS}}-V_{\mathrm{GS}}$ curves in Fig. 5 as a function of drain current (Pix. 4 and 7).

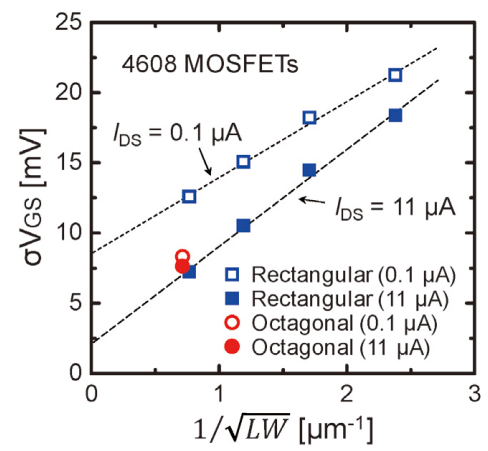

Fig. 9 Standard deviation of $V_{\mathrm{GS}}$ in rectangular and octagonal SF transistors as a function of $(L W)^{-1 / 2}$ (Pix. 1-4 and 7)

rectangular SF transistors (Pix. 1-4) as a function of $(L W)^{-1 / 2}$. Here, the gate width of the octagonal SF transistor uses the average gate width as shown in Fig. $2\left(W_{\text {Ave }}\right)$. Focusing on the distribution of the rectangular $\mathrm{SF}$ transistors, it is clear that the $\sigma V_{\mathrm{GS}}$ increases linearly with respect to $\left.\left.(L W)^{-1 / 2} 20\right)-27\right)$. From this tendency, it is considered that the variation of $V_{\mathrm{GS}}$ is suppressed in the octagonal SF transistors especially in the low drain current condition, compared with the rectangular SF transistors. Furthermore, by focusing on the intercept of the fitting line of the data, the offset of $\sigma V_{\mathrm{GS}}$ becomes large when the drain current decreases.

From the obtained results, it is considered that the difference of the noise characteristics between the octagonal and the rectangular SF transistors would be caused by the parasitic transistor due to the sub channel around the STI edge. As shown in Fig. 10, electric field concentration would occur at the STI edge, and percolation of channel ${ }^{28)-31)}$ becomes significant in that area. This is the reason why the SS and the variation of SS become larger in the rectangular SF transistors than the octagonal transistors. Moreover, the trap density at the STI edge would be higher than that at the gate insulator film on main channel because the appearance probability of RTN in rectangular SF transistors 


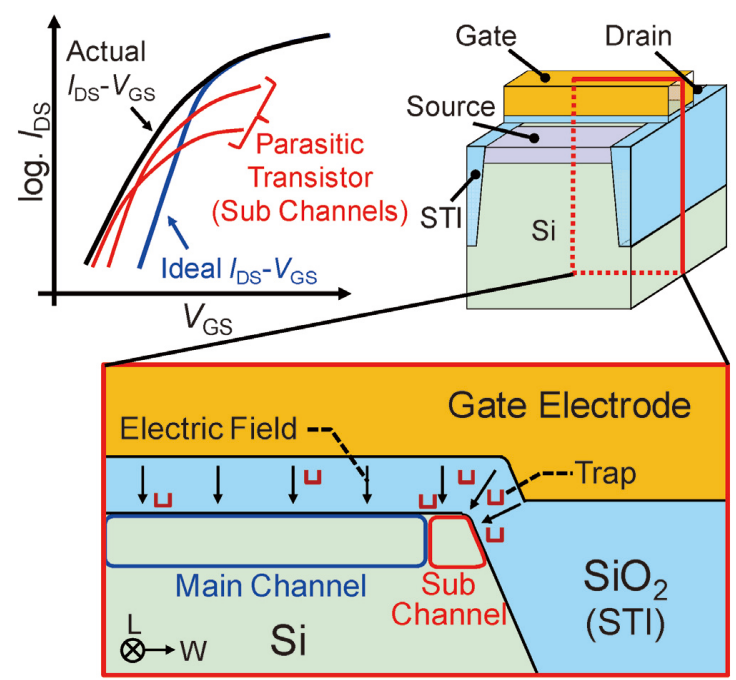

Fig. 10 Schematic illustration of the influence of parasitic transistor due to the sub channel formed by electric field concentration around STI edge.

becomes larger than that of the octagonal SF transistors at the same range of SS as shown in Fig. 7. Based on these results, it is considered that for rectangular SF transistors the interaction between the percolation path and the traps becomes significant at low drain current where the sub channel dominates the drain current. Therefore, there was a large difference in noise distribution between the octagonal and the rectangular SF transistors at the low drain current condition as shown in Fig. 4. On the other hand, the octagonal transistor is also effective shape for reducing the variation of $V_{\mathrm{GS}}$. It is considered that the influence of the sub channel around the STI edge of the rectangular transistors appears as an increase in the offset of $\sigma V_{\mathrm{GS}}$ significantly, especially at low drain current condition. Based on the above discussion, the SF transistor without STI edge can suppress the appearance probability of $\mathrm{RTN}$ and the $V_{\mathrm{GS}}$ variation.

As next, the evaluation of the trapezoidal SF transistors which have an asymmetry gate width at source and drain side is discussed. Figure 11 shows the cumulative probability of $V_{\text {RMS }}$ of the two types of the trapezoidal SF transistor in the Gumbel plot under the drain current of $0.1 \mu \mathrm{A}, 1 \mu \mathrm{A}$ and the $11 \mu \mathrm{A}$, respectively. The SF output voltages were set at $1.9 \mathrm{~V}$ for each type of transistor for the same reason as the evaluation of the octagonal SF transistors. From the distributions in Fig. 11, the trapezoidal SF transistors with the narrower gate width at source side tend to exhibit smaller $V_{\text {RMS }}$ values than those of the wider ones under the constant drain current conditions. This relationship does not change depending on the drain current conditions (from $0.1 \mu \mathrm{A}$ to $11 \mu \mathrm{A}$ in this work). Figure 12 shows the cumulative probability of the amplitude of two states RTN of two types of the trapezoidal SF transistors in the Gumbel plot under the drain current of $0.1 \mu \mathrm{A}, 1 \mu \mathrm{A}$ and $11 \mu \mathrm{A}$, respectively. Here, it is considered that two states RTN is induced by a single trap, and three or more states RTN are induced by multiple traps ${ }^{32}$. The number of RTN states is extracted from counting the peak of the voltage histogram, and the RTN amplitude is defined as a difference between two normal distributions in a voltage histogram of the output time waveform ${ }^{33}$. There were some SF transistors that showed three or more states RTN induced by multiple traps in the experiments. However, the proportion of three or more states RTN to the total cells with RTN was less than $10 \%$ under all the measured drain current conditions. Therefore, in this work, we focused on the amplitude of the most fundamental two states RTN induced by capture or emission of carriers by a single trap, and the detection limit of RTN amplitude was set at $130 \mu \mathrm{V}$. From these distributions in Fig. 12, it is clear that the appearance probability of RTN with large amplitude becomes small
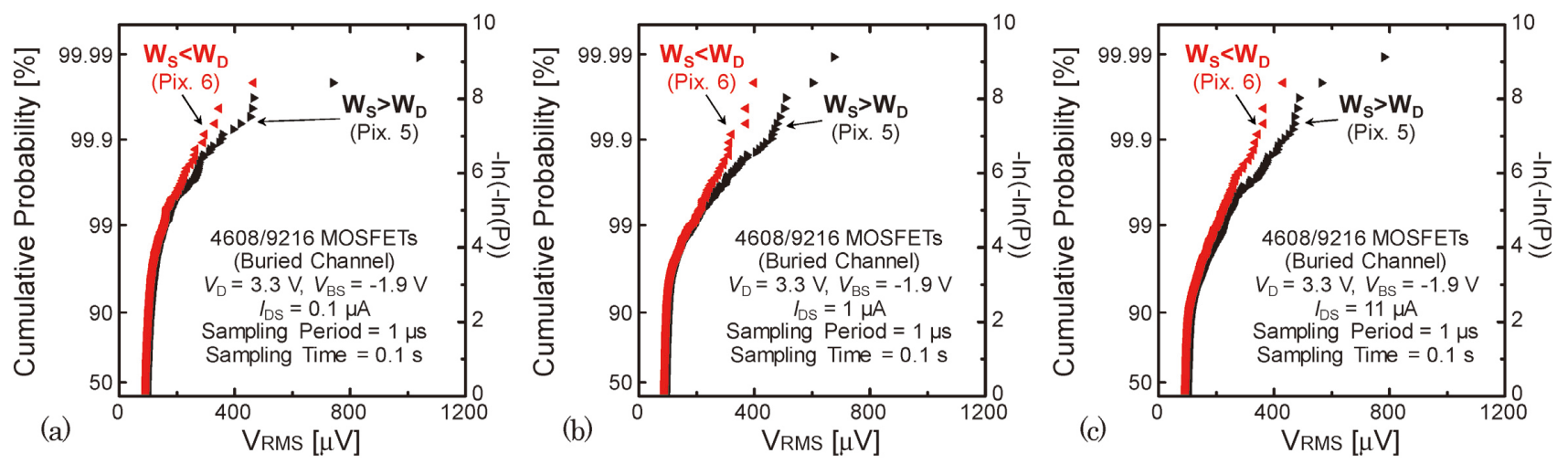

Fig. 11 Cumulative probability of $V_{\mathrm{RMS}}$ in the Gumbel plot (Pix. 5 and 6). The comparison between two types of trapezoidal SF transistor under the constant drain current of (a) $0.1 \mu \mathrm{A}$, (b) $1 \mu \mathrm{A}$ and (c) $11 \mu \mathrm{A}$ is shown. 

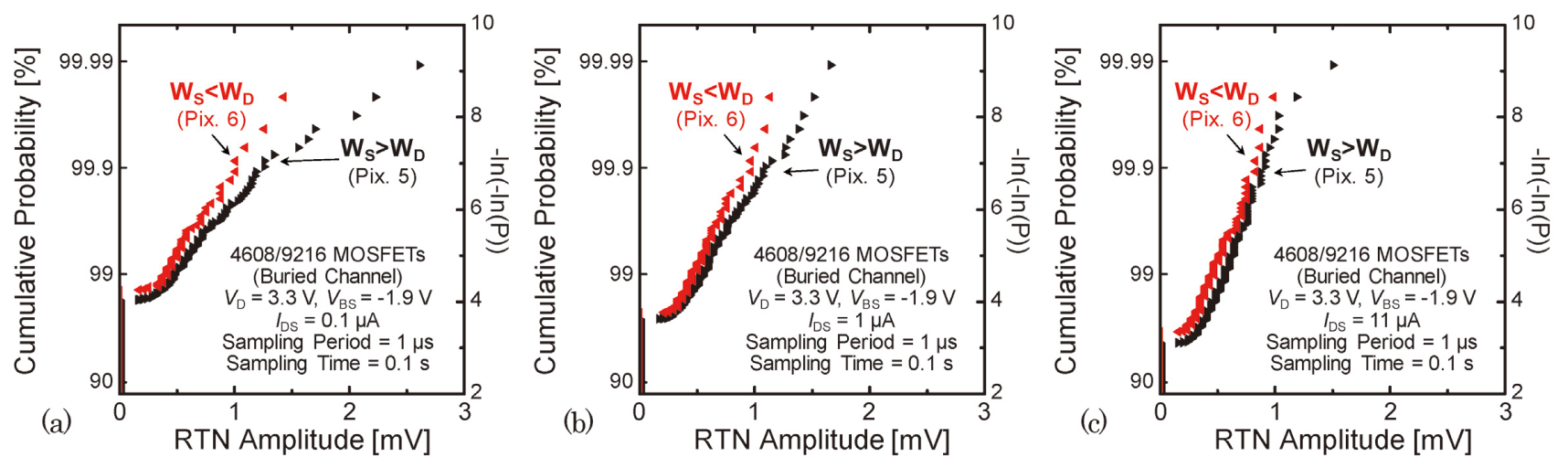

Fig. 12 Cumulative probability of the amplitude of two states RTN in the Gumbel plot (Pix. 5 and 6). The comparison between two types of trapezoidal SF transistor under the constant drain current of (a) $0.1 \mu \mathrm{A}$, (b) $1 \mu \mathrm{A}$ and (c) $11 \mu \mathrm{A}$ is shown.

(a)

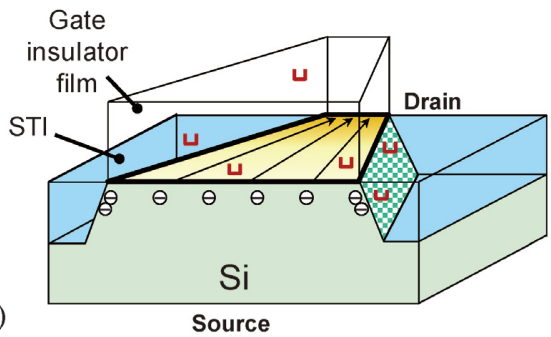

(b)

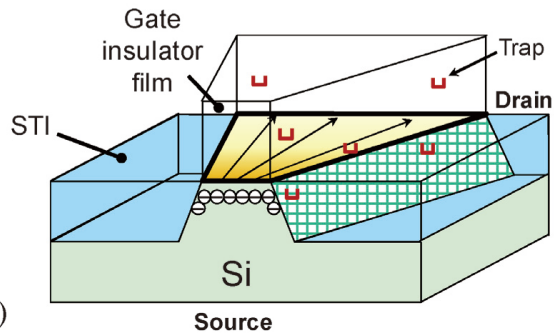

Fig. 13 Schematic illustrations of the trapezoidal SF transistors. (a) Trapezoidal SF transistor with wider gate width at source side (Pix. 5) and (b) trapezoidal SF transistor with narrower gate width at source side (Pix. 6).

in the trapezoidal SF transistors with narrower gate width at source side under the constant drain current conditions. These distributions show the same tendency as the distributions of the $V_{\text {RMS }}$ in Fig. 11. For this reason, it is considered that the difference of the distribution of $V_{\mathrm{RMS}}$ in two types of the trapezoidal $\mathrm{SF}$ transistors is due to the difference of the distribution of the RTN amplitude ${ }^{15}$.

From the obtained results in the evaluation of the trapezoidal SF transistors, we consider the reduction effect of RTN. Figure 13 shows the schematic illustrations of the two types of the trapezoidal SF transistors. It has been reported that the traps at the source side have a larger influence on the appearance of RTN than those at the drain side ${ }^{18)}$. In addition, it is clarified that RTN with large $V_{\text {RMS }}$ decreases statistically as the drain current becomes larger in the same size of SF transistors because the influence of the charged trap reduces at the high carrier density condition $^{12) 15)}$. Moreover, the amplitude of RTN becomes smaller at the larger drain current condition because the percolated channel path becomes wider ${ }^{34) 35)}$. For these reasons, it is considered that the influence of $\mathrm{RTN}$ becomes smaller in the trapezoidal SF transistors with narrower gate width at source side under the constant drain current condition because the carrier density at the source side which greatly influences the appearance of RTN increases and it reduces the RTN amplitude. However, it is necessary to pay attention that the region around the STI edge in the gate width at the source side relatively increases when the gate width at this side is narrow. Furthermore, it is also necessary to pay attention that the appearance probability of RTN with small amplitude increases as the drain current increases especially in the region where the $V_{\mathrm{RMS}}$ is small ${ }^{15)}$.

From these statistical analyses of RTN with SF transistor with various gate shapes, it is considered that the transistor shape which the channel carriers are not influenced by the STI edge ${ }^{36) 37)}$ and the narrower gate width at source side is effective for reducing RTN which has large $V_{\text {RMS }}$.

\section{Conclusion}

In this paper, the statistical analysis of RTN in SF with various transistor gate shapes by using the array test circuit was discussed. From the evaluation of the octagonal SF transistors which have no STI edge, it is considered that the transistor structure without STI edge is effective for reducing RTN and variation of $V_{\mathrm{GS}}$ because of the suppression of the generation of percolated channel around STI edge where the trap density is likely to be high. In addition, from the evaluation of the trapezoidal SF transistors which have 
an asymmetry gate width at source and drain side, devices for increasing the carrier density at the source side are effective for reducing RTN with large magnitude because the influence of the charged trap which significantly affects the appearance of RTN reduces. These findings obtained by the statistical analysis of RTN can give a guidance to the design of inpixel SF transistors with small RTN for low noise CIS and further evaluation of RTN behavior is expected to be more important from now on.

\section{Acknowledgment}

This work was partly supported by JSPS KAKENHI Grant Number 15H62245 and 17H04921.

\section{References}

1) M.J. Kirton and M.J. Uren: "Noise in solid-state microstructures - a new perspective on individual defects, interface states and lowfrequency (1/f) noise", Adv. Phys. 38, pp.367-468 (1989)

2) C. Leyris, F. Martinez, M. Valenza, A. Hoffmann, J.C. Vildeuil and F. Roy: "Impact of Random Telegraph Signal in CMOS Image Sensors for Low-Light Levels", Solid State Circuits Conf., pp.376379 (2006)

3) X. Wang, P.R. Rao, A. Mierop and A.J. P. Theuwissen: "Random Telegraph Signal in CMOS Image Sensor Pixels", IEDM Tech. Dig., pp.115-118 (2006)

4) J.Y. Kim, S.I. Hwang, J.J. Lee, J.H. Ko, Y. Kim, J.C. Ahn, T. Asaba and Y.H. Lee: "Characterization and improvement of random noise in 1/3.2" UXGA CMOS image sensor with 2.8 um pixel using 0.13um-technology", IEEE Work. CCDs AIS. Japan, pp.149-152 (2005)

5) X. Wang, M.F. Snoeij, P.R. Rao, A. Mierop and A.J. P. Theuwissen: "A CMOS Image Sensor with a Buried-Channel Source Follower", Int. Solid-State Circuits Conf., pp.62-64 (2008)

6) H. Suzuki, R. Kuroda, A. Teramoto, A. Yonezawa, S. Sugawa and T. Ohmi: "Impact of Random Telegraph Noise Reduction with Buried Channel MOSFET", Int. Conf. Solid State Devices and Materials, pp.851-852 (2011)

7) A. Yonezawa, A. Teramoto, R. Kuroda, H. Suzuki, S. Sugawa and T. Ohmi: "Statistical Analysis of Random Telegraph Noise Reduction Effect by Separating Channel From the Interface", Int. Reliability Physics Symp., 3B.5.1 (2012)

8) T. Goto, R. Kuroda, N. Akagawa, T. Suwa, A. Teramoto, X. Li, T. Obara, D. Kimoto, S. Sugawa, T. Ohmi, Y. Kamata, Y. Kumagai and K. Shibusawa: "Atomically flattening of Si surface of silicon on insulator and isolation-patterned wafers", Jpn.J. Appl. Phys. 54, 04DA04 (2015)

9) D. Pates, J.-H. Lyu, S. Osawa, I. Takayanagi, T. Sato, T. Bales, K. Kawamura, E. Pages, S. Matsuo, T. Kawaguchi, T. Sugiki, N. Yoshimura, J. Nakamura, J. Ladd, Z. Yin, R. Iimura, X. Fan, S. Johnson, A. Rayankula, R. Mauritzson and G. Agranov: "An APS-C Format 14b Digital CMOS Image Sensor with a Dynamic Response Pixel", Dig. Tech. Pap. Int. Solid-State Circuits Conf. 43, pp.418420 (2011)

10) M.W. Seo, S. Kawahito, K. Yasutomi, K. Kagawa and N. Teranishi: "A Low Dark Leakage Current High-Sensitivity CMOS Image Sensor With STI-Less Shared Pixel Design", IEEE Trans. Electron Devices 61, pp.2093-2097 (2014)

11) S. Ichino, T. Mawaki, S. Wakashima, A. Teramoto, R. Kuroda, P. Gaubert, T. Goto, T. Suwa and S. Sugawa: "Statistical Analysis of Random Telegraph Noise in Source Follower Transistors with Various Shapes", Int. Image Sensor Workshop, pp.39-42 (2017)
12) K. Abe, S. Sugawa, R. Kuroda, S. Watabe, N. Miyamoto, A. Teramoto, T. Ohmi, Y. Kamata and K. Shibusawa: "Analysis of Source Follower Random Telegraph Signal Using nMOS and pMOS Array TEG", Int. Image Sensor Workshop, pp.62-65 (2007)

13) T. Mawaki, A. Teramoto, R. Kuroda, S. Ichino and S. Sugawa: "Analysis of Random Telegraph Noise Behaviors of nMOS and pMOS toward Back Bias Voltage Changing", Int. Conf. Solid State Devices and Materials, pp.333-334 (2017)

14) A. Yonezawa, A. Teramoto, T. Obara, R. Kuroda, S. Sugawa and T. Ohmi: "The Study of Time Constant Analysis in Random Telegraph Noise at the Subthreshold Voltage Region", Int. Reliability Physics Symp., XT.11.1 (2013)

15) S. Ichino, T. Mawaki, A. Teramoto, R. Kuroda, H. Park, T. Maeda, S. Wakashima, T. Goto, T. Suwa and S. Sugawa: "Impact of Drain Current to Appearance Probability and Amplitude of Random Telegraph Noise in Low Noise CMOS Image Sensors", Int. Conf. Solid State Devices and Materials, pp.331-332 (2017)

16) K.S. Ralls, W.J. Skocpol, L.D. Jackel, R.E. Howard, L.A. Fetter, R.W. Epworth and D.M. Tennant: "Discrete Resistance Switching in Submicrometer Silicon Inversion Layers: Individual Interface Traps and Low-Frequency (1/f) Noise", Phys. Rev. Lett. 52, pp.228231 (1984)

17) T. Nagumo, K. Takeuchi, S. Yokogawa, K. Imai and Y. Hayashi: "New Analysis Methods for Comprehensive Understanding of Random Telegraph Noise", IEDM Tech. Dig., pp.759-762 (2009)

18) K. Abe, Y. Kumagai, S. Sugawa, S. Watabe, T. Fujisawa, A. Teramoto and T. Ohmi: "Asymmetry of RTS Characteristics along Source-Drain Direction and Statistical Analysis of Process-Induced RTS", Int. Reliability Physics Symp., pp.996-1001 (2009)

19) R. Kuroda, A. Yonezawa, A. Teramoto, T.-L. Li, Y. Tochigi and S. Sugawa: "A Statistical Evaluation of Random Telegraph Noise of In-Pixel Source Follower Equivalent Surface and Buried Channel Transistors", IEEE Trans. Electron Devices 60, pp.3555-3561 (2013)

20) M.J. M. Pelgrom, A.C. J. Duinmaijer and A.P. G. Welbers: "Matching Properties of MOS Transistors", IEEE J. Solid-State Circuits 24, pp.1433-1440 (1989)

21) T. Mizuno, J. Okamura and A. Toriumi: "Experimental Study of Threshold Voltage Fluctuation Due to Statistical Variation of Channel Dopant Number in MOSFET"s", IEEE Trans. Electron Devices 41, pp.2216-2221 (1994)

22) M. Eisele, J. Berthold, R. Thewes, E. Wohlrab, D. SchmittiLandsiedel and W. Weber: "Intra-Die Device Parameter Variations and Their Impact on Digital CMOS Gates at Low Supply Voltages", IEDM Tech. Dig., pp.67-70 (1995)

23) K. Takeuchi, T. Tatsumi and A. Furukawa: "Channel Engineering for the Reduction of Random-Dopant-Placement-Induced Threshold Voltage Fluctuation", IEDM Tech. Dig., pp.841-844 (1997)

24) A. Mizunuma, T. Oishi, N. Yokoyama, M. Nonaka, S. Tanaka and H. Ammo: "Study of 90-nm MOSFET Subthreshold Hump Characteristics Using Newly Developed MOSFET Array Test Structure", IEEE Trans. Semicond. Manuf. 19, pp.19-26 (2006)

25) S. Watabe, S. Sugawa, A. Teramoto and T. Ohmi: "New Statistical Evaluation Method for the Variation of Metal-OxideSemiconductor Field-Effect Transistors", Jpn.J. Appl. Phys. 46, pp.2054-2057 (2007)

26) K. Abe, S. Sugawa, S. Watabe, N. Miyamoto, A.Teramoto, Y. Kamata, K. Shibusawa, M. Toita and T. Ohmi: "Random Telegraph Signal Statistical Analysis using a Very Large-scale Array TEG with 1M MOSFETs", Symp. VLSI Technology, pp.210211 (2007)

27) K. Abe, A. Teramoto, S. Watabe, T. Fujisawa, S. Sugawa, Y. Kamata, K. Shibusawa and T. Ohmi: "Experimental Investigation of Effect of Channel Doping Concentration on Random Telegraph Signal Noise", Jpn.J. Appl. Phys. 49, 04 DC07 (2010)

28) H.H. Mueller and M. Schulz: "Random telegraph signal: An atomic probe of the local current in field-effect transistors", J. Appl. Phys. 83, pp.1734-1741 (1998) 
29) H. -S. Wong and Y. Taur: "Three-Dimensional "Atomic" Simulation

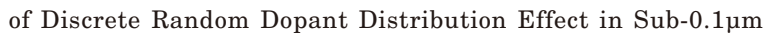
MOSFETs", IEDM Tech. Dig., pp.705-708 (1993)

30) A. Asenov, R. Balasubramaniam, A.R. Brown and J.H. Davies: "RTS Amplitudes in Decananometer MOSFETs: 3-D Simulation Study", IEEE Trans. Electron Devices 50, pp.839-845 (2003)

31) K. Sonoda, K. Ishikawa, T. Eimori and O. Tsuchiya: "Discrete Dopant Effects on Statistical Variation of Random Telegraph Signal Magnitude", IEEE Trans. Electron Devices 54, pp.19181925 (2007)

32) T. Obara, A. Teramoto, A. Yonezawa, R. Kuroda, S. Sugawa and T. Ohmi: "Analyzing Correlation between Multiple Traps in RTN Characteristics", Int. Reliability Physics Symp., 4A.6.1 (2014)

33) K. Abe, A. Teramoto, S. Sugawa and T. Ohmi: "Understanding of Traps Causing Random Telegraph Noise Based on Experimentally Extracted Time Constants and Amplitude", Int. Reliability Physics Symp., 4A.4.1 (2011)

34) A. Asenov, A.R. Brown, J.H. Davies, S. Kaya and G. Slavcheva: "Simulation of Intrinsic Parameter Fluctuations in Decananometer and Nanometer-Scale MOSFETs", IEEE Trans. Electron Devices 50, pp.1837-1852 (2003)

35) P. Ren, P. Hao, C. Liu, R. Wang, X. Jiang, Y. Qiu, R. Huang, S. Guo, M. Luo, J. Zou, M. Li, J. Wang, J. Wu, J. Liu, W. Bu, W. Wong, S. Yu, H. Wu, S. -W. Lee and Y. Wang, "New Observations on Complex RTN in Scaled High-k/Metal-gate MOSFETs - the Role of Defect Coupling under DC/AC Condition", IEDM Tech. Dig., pp.778-781 (2013)

36) P. Martin-Gonthier, E. Havard and P. Magnan: "Custom transistor layout design techniques for random telegraph signal noise reduction in CMOS image sensors", Electronics Letters 46, no. 19 (2010)

37) K. Itonaga, K. Mizuta, T. Kataoka, M. Yanagita, H. Ikeda, H. Ishiwata, Y. Tanaka, T. Wakano, Y. Matoba, T. Oishi, R. Yamamoto, S. Arakawa, J. Komachi, M. Katsumata, S. Watanabe, S. Saito, T. Haruta, S. Matsumoto, K. Ohno, T. Ezaki, T. Nagano and T. Hirayama: "Extremely-Low-Noise CMOS Image Sensor with High Saturation Capacity", IEDM Tech. Dig., pp.171-174 (2011)

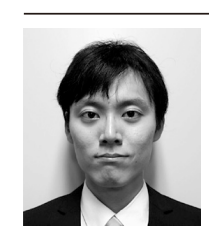

Shinya Ichino received the B.S. degree in electronic engineering from Tohoku University, Sendai, Japan, in 2017, where he is currently working toward the M.S. degree at the Graduate School of Engineering, Tohoku University. His current research topics are measurement and analyses of random telegraph noise in CMOS image sensor.

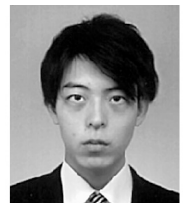

Takezo Mawaki received the B.S. degree in electronic engineering and the M.S. degree in management science and technology from Tohoku University, Sendai, Japan, in 2016 and 2018, respectively. He is currently pursuing the $\mathrm{Ph} . \mathrm{D}$. degree at the Graduate School of Engineering, Tohoku University. His current research topics are measurement and analyses of random telegraph noise.

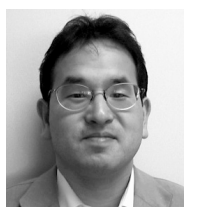

Akinobu Teramoto received the B.S., M.S. and $\mathrm{Ph} . \mathrm{D}$. degrees in electronic engineering from Tohoku University, Sendai, Japan, in 1990, 1992, and 2001, respectively. In 1992-2002, he was with Mitsubishi Electric Corp. In 2002, he moved to Tohoku University, where he is currently a Professor with the New Industry Creation Hatchery Center.

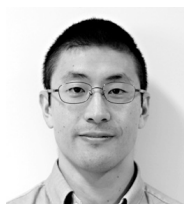

Rihito Kuroda received the B.S. degree in electronic engineering and the M.S. and Ph.D. degrees in management science and technology from Tohoku University, Sendai, Japan, in 2005, 2007, and 2010, respectively. He was a Research Fellow of the Japan Society for the Promotion of Science Research from 2007 to 2010. Since 2010, he is with the Graduate School of Engineering, Tohoku University, where he is currently an Associate Professor. ITE member.

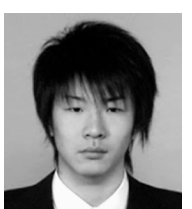

Shunichi Wakashima received the B.S. degree in electronic engineering and the M.S. and $\mathrm{Ph} . \mathrm{D}$. degrees in management science and technology from Tohoku University, Sendai, Japan, in 2012, 2014 and 2017, respectively. Since 2017, he is with Canon Inc.

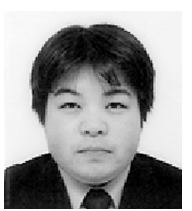

Tomoyuki Suwa received the B.S., M.S. and Ph.D. degrees in electronic engineering from Tohoku University, Sendai, Japan, in 2001, 2003, and 2006 respectively. He is currently an Associate Professor in the New Industry Creation Hatchery Center, Tohoku University.

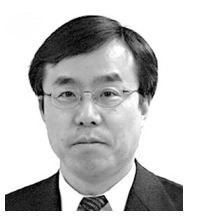

Shigetoshi Sugawa received the M.S. degree in physics from the Tokyo Institute of Technology, Tokyo, Japan, in 1982 and the Ph.D. degree in electronic engineering from Tohoku University, Sendai, Japan, in 1996. In 1982-1999, he was with Canon Inc. In 1999, he moved to Tohoku University, where he is currently a Professor with the Graduate School of Engineering, also with the New Industry Creation Hatchery Center. ITE fellow. 